\title{
Partitioning Cursor Movements in "Point and Click" Tasks
}

\author{
Faustina Hwang \\ University of Cambridge, Department of Engineering \\ Trumpington Street, Cambridge, UK CB2 1PZ \\ Tel: +44 1223766958 Email: fh220@cam.ac.uk
}

\begin{abstract}
Studies of cursor trajectories can help explain performance differences in "point and click" tasks. As users can have different difficulties with moving the cursor to a point on the screen, as compared with pressing a button to select an object, it is helpful to study the two stages of the interaction separately. This paper proposes a method of partitioning a cursor trajectory into a travel and a select phase. The movements of motion-impaired users are studied to show that, by analyzing the two phases separately, it is possible to capture aspects of movement that are otherwise lost.
\end{abstract}

\section{Keywords}

Cursor trajectories, motion-impaired users, pointing devices

\section{INTRODUCTION}

"Point and click" interactions using a pointing device are key features of graphical user interfaces (GUIs). Task performance, however, can differ between input devices or between user capabilities. For example, motion-impaired users can often have difficulty with accurate control of standard pointing devices [4], resulting in poorer performance as compared with able-bodied users.

A full understanding of why such performance differences arise can facilitate the design of interfaces and input devices that are better suited to user needs. Studies of cursor movement have traditionally evaluated performance in terms of task completion times and error rates. Although these measures can show that differences exist between conditions, establishing why they exist is more likely to be accomplished by analyzing the path of movement throughout a trial [2].

Analyses of the cursor's path of movement have tended to examine the "point and click" trajectory as a whole [e.g. 2, 5]. However, users may experience separate difficulties with moving the cursor to a particular point on the screen, as compared with pressing a button to select an object, and this distinction may be lost when the trajectory is considered as a whole. For example, MacKenzie et al [2] define a measure that counts the number of target re-entries (TREs) throughout a trial. However, no distinction is made between TREs that occur as a result of the cursor "shooting" through the target, and those that occur as a result of the cursor slipping off the target during selection. These two types of TREs are indicative of different difficulties that may require distinct remedies, suggesting that there is benefit to be gained from partitioning the cursor movement into phases, and studying each separately.

In the past, different methods of partitioning cursor trajectories in "point and click" tasks have been employed. For example, Akamatsu et al [1] extracted the cursor's "final positioning time," i.e. the time to complete the trial once the cursor entered the target region, from the total positioning time. Phillips and Triggs [3] studied cursor movements in terms of an accelerative and a decelerative phase, where the transition between the two was deemed to occur when the cursor reached peak velocity. However, these methods may not always be appropriate, as in the analysis of trajectories of motion-impaired users where, for example, a spasm may cause the cursor to pass through the target region at a high velocity very early on in the task.

This paper proposes a new partitioning method that separates a cursor trajectory into a travel and a select phase and shows that by analyzing each phase separately, it is possible to capture aspects of cursor movement that are lost when the movement is considered as a whole.

\section{MOVEMENT PARTITIONING}

"Point and click" interactions with a mouse require a user to move the cursor to a particular target on the screen, then to press and release a button while holding the cursor inside the target. The travel and select phases are intended to represent these two stages of the interaction.

To define the two phases, a cursor trajectory is first divided into submovements using methods similar to those described in [5]. A submovement is defined to begin when $20 \mathrm{~ms}$ of cursor motion with a speed greater than zero occurs. The submovement is considered to be over either when the speed returns to zero, or when the acceleration changes from negative to positive while the speed is less than half the peak. In the latter case, the next submovement is defined to begin at the end of the previous one.

Defining $S 1$ to be the first submovement to end while the cursor is inside the target, the transition from the travel phase to the select phase is defined occur at the end of S1. 


\section{STUDYING THE MOVEMENT PHASES}

In this section, the movements of three motion-impaired computer users are examined to illustrate the potential benefits of studying cursor trajectories in terms of separate travel and select phases.

As part of a larger study, users PI3, PI6, and PI7 performed a series of multidirectional "point and click" interactions using a Logitech Wingman force-feedback mouse for input. 16 "target" circles (diameter $=12 \mathrm{~mm}$ ) were arranged in a circular layout (diameter $=172 \mathrm{~mm}$ ) around a central "home" circle. The participants selected each of the 16 "targets", alternating "target" selections with "home" selections. PI3 had Cerebral Palsy and exhibited frequent spasms in his dominant arm. PI7 had Friedrich's Ataxia and exhibited a constant tremor in both hands and arms. In addition, PI7 performed the "point and click" task with two hands, using one hand to navigate the mouse and the other to press the left mouse button for selection. In contrast, PI6 had only a mild impairment in his dominant hand and arm.

The mean task completion times for PI3 and PI7 were approximately 6 and 5 times longer respectively than that for PI6 (Figure 1). A higher number of total target re-entries [2] (Figure 2) suggests that part of this time increase can be attributed to difficulties with positioning the pointer inside the target and keeping it there. It is of particular interest, however, to note that both PI3 and PI7 exhibit similar numbers of target re-entries, despite having dissimilar cursor behaviors. PI3 experiences spasms which often cause the cursor to "shoot through" the target as well as to slip off the target during selection. In contrast, PI7 has less trouble positioning the cursor inside the target, but has much greater difficulty with clicking the mouse button and holding the cursor steady at the same time.

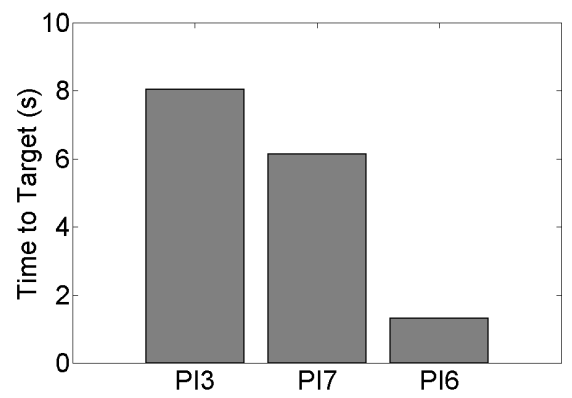

Figure 1. Task completion times.

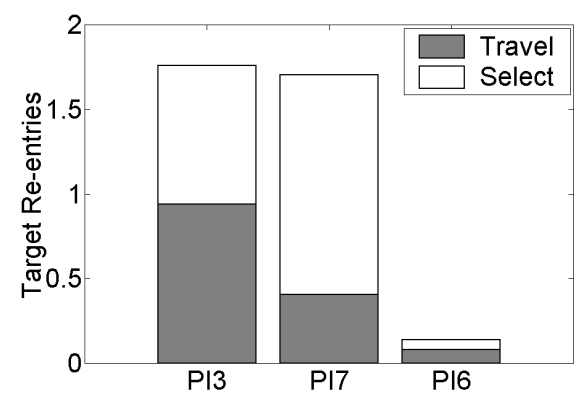

Figure 2. Target re-entries for the travel and select phases.
Although the total number of target re-entries does not differentiate between these two problem areas, by considering the travel and select phases separately, it is possible to capture this difference. PI3 exhibits a higher number of target re-entries during the travel phase than PI7 (Figure 2), reflecting a greater difficulty with getting the cursor to stop inside the target. PI7 exhibits a much higher proportion of target re-entries during the select phase as compared with the travel phase, thereby capturing repeated cycles of clicking the mouse button, slipping off the target, and repositioning the mouse.

\section{IMPLICATIONS FOR INTERFACE DESIGN}

Partitioning the cursor movement into travel and select phases and analyzing the two phases separately gives an increased ability to identify and discriminate among cursor behaviors. A more thorough understanding of cursor movements, particularly areas of difficulty, can facilitate the design of interfaces that are better suited to user needs by providing insight about ways to improve the interaction, be it through better device design or more appropriate matching of users with forms of assistance. For example, a high number of target re-entries in the select phase suggests that PI7 may benefit from using dwell selection which would remove the necessity for a button click and consequently reduce the number of target re-entries. On the other hand, dwell selection is likely to be of less benefit to $\mathrm{PI} 3$, as it does not provide any assistance with positioning the cursor inside the target..

\section{ACKNOWLEDGMENTS}

The author thanks S. Keates, P. Langdon, and J. Clarkson for their guidance. Many thanks also to the volunteers and staff of the Papworth Trust. This work is funded by the Canadian Cambridge Trust and NSERC.

\section{REFERENCES}

1. Akamatsu, M., MacKenzie, I.S., and Hasbrouq, T. A comparison of tactile, auditory, and visual feedback in a pointing task using a mouse-type device, Ergonomics 38 (1995), 816-827.

2. MacKenzie, I. S., Kauppinen, T., and Silfverberg, M. Accuracy measures for evaluating computer pointing devices. In Proceedings of CHI 2001 (Seattle WA, 2001), ACM Press, 9-15.

3. Phillips, J.G. and Triggs, T.J. Characteristics of cursor trajectories controlled by the computer mouse, Ergonomics 44(5), (2001), 527-536.

4. Trewin, S. and Pain, H. Keyboard and mouse errors due to motor disabilities, International Journal of HumanComputer Studies 50, 2 (1999), 109-144

5. Walker, N., Meyer, D.E., and Smelcer, J.B. Spatial and temporal characteristics of rapid cursor-positioning movements with electromechanical mice in humancomputer interaction, Human Factors 35(3), (1993), 431-458. 